ARTÍCULO DE REFLEXIÓN

\title{
Lo sagrado y la religión en la sociedad moderna
}

The sacred and religion in modern society

\author{
William Oswaldo Aparicio Gómez ${ }^{1}$ \\ $E d \& T I C$
}

Recibido: 01.11.2020

Aceptado: 15.11.2020

\section{Resumen}

El presente artículo se basa en el libro titulado "Para comprender las nuevas formas de la religión” (Mardones, 1998), y sus reflexiones sobre los principales aspectos de lo sagrado desde una mirada fenomenológica, el concepto de religión desde una perspectiva más sociológica, y la distinción de tipo conceptual entre lo sagrado y la religión.

Palabras clave: Ciencias de la Educación, Religión, Filosofía, Ética, Naturaleza humana, Ontología.

\begin{abstract}
This article is based on the book entitled "To understand the new forms of religion" (Mardonés, 1998), and his reflections on the main aspects of the sacred from a
\end{abstract}

\footnotetext{
${ }^{1}$ waparicio@editic.net https://orcid.org/0000-0002-8178-1253

https://scholar.google.com/citations?user=V5RCdbUAAAAJ\&hl=es\&oi=ao
} 
phenomenological point of view, the concept of religion from a more sociological point of view, and the conceptual distinction between the sacred and religion.

Keywords: Education Sciences, Religion, Philosophy, Ethics, Human Nature, Ontology.

\section{La experiencia de lo sagrado}

Podemos hacer una primera aproximación a lo sagrado analizando tres grandes perspectivas: sociológica, fenomenológica y hermenéutica.

- En la perspectiva sociológica lo sagrado aparece como un mero producto de la sociedad.

- En la perspectiva fenomenológica se destacan tres aspectos de lo sagrado: la presencia de lo numinoso o de la conciencia particular de la experiencia religiosa como poder oculto y misterioso, la expresión natural de la santidad de lo numinoso como algo majestuoso y tremendo, y, por último, la fascinación y atracción con que se manifiesta lo numinoso.

- En la perspectiva hermenéutica, se intenta integrar y articular las diversas aportaciones y métodos de las disciplinas que abordan el estudio del fenómeno religioso tales como la historia, la sicología, la historia y la etnología; se parte de que lo sagrado es una realidad absoluta que trasciende el mundo, pero que se manifiesta en él por medio del símbolo, el cual permite al hombre captar y comunicar lo sagrado.

Dejadas las anteriores perspectivas se hace imprescindible hacer un análisis semántico de las expresiones lingüísticas de lo sagrado y las diferentes traducciones, incorporando también el término misterio y divinidad para referirse a lo sagrado.

Detrás de la experiencia de lo sagrado está la búsqueda y el desvelamiento de la realidad en su hondura más abismática, y la única forma como se revela en el mundo es por medio de los 
símbolos; en otras palabras, que las hierofanías (manifestación de lo sagrado en el mundo de lo profano) se hacen accesibles a la humanidad simbólicamente.

El símbolo es entonces la mediación de lo externo en lo temporal; la raja, el resquicio, la apertura por la cual se cuelan en nuestro mundo presente las fuentes profundas de la vida, es un lenguaje de la evocación y la excedencia (Mardones, 1998). Solo por medio de él podemos captar y comunicar con lo sagrado (Lamo de Espinosa Michels de Champourcin, 2009).

Desde este contexto el autor introduce el tema del mito como aquel que permite explicar el dinamismo del símbolo (Sánchez Jerez, 2007). El mito es un relato especial: apunta a esa realidad profunda, última, numinosa y ejerce a su vez una serie de funciones sociales: es el que abre a la dimensión de misterio presente en el mundo haciendo de vehículo para que se haga accequible lo sagrado, tiene una función cosmológica al permitir a la ciencia explicar el mundo, fundamenta y valida un cierto orden social (Zuidema, 2020), y por último tiene una función pedagógica pues enseña a vivir la vida humana desde cualquier circunstancia (Durkheim, 1968).

El mito tiene su continuidad en los ritos pues tanto ellos como las ceremonias, son algo así como espectáculos rituales en los que se representa un episodio mitológico. El rito tiene carácter repetitivo donde aparecen símbolos y mitos (Paiva et al., 2004), de ahí que (como toda acción simbólica) le pertenece:

- En primer lugar, desde el punto de vista epistemológico, la multivocidad, la pluralidad de significaciones y evocaciones, el ser un conocimiento que llama a la participación y es, por tanto, implicativo.

- En segundo lugar, el carácter evocador y celebrativo de lo numinoso que busca transformar la banalidad de la vida ordinaria, de ahí la repetición, el rito es acción hierótica simbólica.

- en tercer lugar, el estar dotado culturalmente de concepciones cosmológicas y valores con fuerza emotiva persuasiva. 
Se hace imprescindible distinguir términos que es bueno precisar para una mejor comprensión de lo sagrado: La trascendencia como aquella dimensión de lo sagrado que está más allá de la experiencia sensible (Abumanssur, 1970), y por tanto indemostrable desde la ciencia, lo divino que hace referencia al mundo sobrenatural de los seres que se supone dotados de la santidad, perfección, poder, lo santo que hace referencia al carácter separado, distinto totalmente respecto a lo mundano y profano.

\section{La sociedad secular}

Lo sagrado es, pues, equivalente a lo poderoso, significativo, viviente y tremendo de la profundidad de lo real. Ahora se hace necesario analizar la sociedad secular (banners, 2017); buscar dónde y cómo aparecen las preocupaciones, búsquedas, temores y fascinaciones de los hombres de hoy para encontrar indicios de lo sagrado en ella. Hay algo eterno en la religión que está destinado a sobrevivir a todos los símbolos particulares con los que sucesivamente se ha recubierto el pensamiento religioso (Durkheim, 1968) y hace que se planteen dos problemas y tareas: por un lado, estar atentos a la evolución de la manifestación de lo sagrado, y por otro, tener claro qué es la sustancia de lo religioso o sagrado en nuestro tiempo. Esta es la tarea que emprende el autor en este segundo apartado.

La sociedad ha ido evolucionando (Diez de Velasco, 2011), y hoy no es extraño afirmar que la religión no está en el centro de la sociedad como ocurrió en tiempos anteriores, incluso ya ni siquiera es vista como importante. Por este motivo ha ido quedando relegada a un segundo plano, ha ido desplazándose hacia la periferia; aparece solo como un ingrediente más en la vida que la persona puede o no tomar. Todo esto ha llevado, a una privatización de la religión en la sociedad actual (Mardones, 1998). La religión ha perdido poder social, pero ha ganado en libertad, pues ya no es vista como un instrumento disciplinar para someter a los individuos de la sociedad (Duch et al., 2008), ya no se mezcla interés político-social con religión, ya no hay una mezcla entre la moralidad pública y la religiosa; y esta situación debe ser aprovechada para que la religión organizada aporte su contribución en la moralidad pública (Mundigo, 
2005). Tal vez la llamada por el autor modernidad secularizada sea una oportunidad para que la religión muestre su lado crítico, desinteresado, y militante en pro del hombre.

Pero este proceso de secularización parece ser un proceso sin fin, donde el hombre está como condenado a construir dioses continuamente y a destruirlos. Desde esta perspectiva podemos hablar de un reencantamiento del mundo en la modernidad (G. \& G., 1994). Se ha dado un desplazamiento de la vivencia religiosa hacia el individuo. Ahora, frente a las exigencias de los dictámenes de la organización se elevan las exigencias del individuo, a este proceso se le llama secularización subjetiva (Mardones, 1998). Ante tantos cambios en la vivencia religiosa se presentan reacciones a las que se tienden: intentar negociar con la situación y adaptarse a ella mediante un proceso de actualización y recuperación de la relevancia social; esto en la práctica se traduce en una línea de comportamiento que va, por un lado, desde el reformismo tenue a las propuestas radicales de cambio institucional y de formulaciones de fe; por otro, desde las resistencias responsables y los escrúpulos para el abandono de determinadas formulaciones, hábitos o prácticas (Aparicio-Gómez, 2016), hasta el rechazo frontal de cualquier adaptación a la modernidad y la huida al ghetto.

La secularización es un factor desestabilizador del orden social y cultural que existía anteriormente. Como afirma el sociólogo M. Weber quien piensa que la modernidad es fruto del cristianismo (Gerth et al., 2013). Hunde sus raíces en la concepción del Dios creador que permite a la creación autonominarze de él y tener su propia consistencia en su finitud. La impulsa la fe en un Dios de la historia, que salva y libera desencadenando una responsabilidad acerca del discurrir político-social. Y ve la ruptura en el hecho de que la secularización parece ser un proceso sin fin prosiguiéndose así un interminable proceso de profanización (Lassman, 2017). Esta secularización, en últimas, lo que ha hecho es romper la continuidad entre lo religiosidad y lo profano (Barros Cantillo, 2015), algo que provoca diversas reacciones:

- El rechazo: lo hacen los nostálgicos que piensan que todo tiempo pasado fue mejor y buscan restaurar desde un cierto integrismo y fundamentalismo lo que ha roto el modernismo. 
- La asimilación: es la actitud de los que buscan un pacto con el proceso de secularización. En el fondo se quisiera asimilar la secularización a la fe cristiana.

- La profundización: es la postura de aquellos que han comprendido que el proceso está clavado en las entrañas del cristianismo y de la modernidad, por tanto, hay que mantener esta tensión sin facilismos ni integrismos, sino construyendo horizontes de sentido a partir de esta ruptura.

Analizando el funcionalismo predominante y su implicación en la religión, es importante notar cómo las condiciones para captar lo sagrado van desapareciendo debido a la mentalidad utilitarista y pragmática de la realidad moderna que hace ver como un valor sólo lo que responde a la razón tecno-científica quitando de en medio a lo sagrado (Mardones, 1998).

La cultura es la forma de la religión (Tillich, 1987), pero ésta es la sustancia de la cultura; con el descentramiento cultural de la religión en la modernidad la sustancia de la cultura moderna, es ahora menos palpable. Aunque el misterio ya no late con tanta fuerza en la cultura, aún así hay que buscar con cuidado en la creatividad artística o científica expresiones de lo sagrado, pues donde corre con fuerza el espíritu humano se trasluce la presencia de un "algo" misteriosos que se refleja pálidamente (Mardones, 1998).

La pregunta de fondo resulta ser: ¿el hombre moderno ha perdido toda comunicación con lo sagrado?, tal vez no, más bien lo sagrado se ha trivializado en expresiones como el horóscopo, astrología, esoterismo, etc. que hacen que se difuminen o se vuelvan menos translúcidas.

\section{Análisis de la modernidad religiosa}

Intentemos precisar el estatuto epistemológico y metodológico de la conceptualización que puede emplearse al hablar de religión. ¿De qué concepción se va a partir para hablar de lo religioso?, ¿de la tradicional que liga lo religioso únicamente a la religión institucionalizada de las grandes iglesias o confesiones?, o ¿de alguna nueva? 
Encontramos cierta limitación de la concepción cristiana de sagrado y religión que se da en todas las respuestas a estas preguntas. Una vez y otra vez, hemos de pasar por la aduana de la concepción cristiana para aceptar si una manifestación es religiosa o no lo es. En la anterior afirmación tal vez existe cierta influencia en E. Durkheim (Mardones, 1998).

Tal vez un intento para obviar este problema es recurrir sólo al concepto de lo sagrado, pero con el riesgo de caer entonces en un gran formalismo y vernos sometidos a la noción de religión institucionalizada y predominante; se hace imprescindible analizar también la salida de las perspectivas analógicas en que se mira lo sagrado en el mundo moderno teniendo cuidado de distinguir entre religión y religiosidad para analizar los nuevos movimientos religiosos (Rivera, 2008), aunque también aquí queda en el fondo la pregunta de si son verdaderamente sagrados los fenómenos religiosos de la modernidad.

Descartado el camino de analizar lo sagrado por no parecer ningún camino real, se hace imprescindible analizar las dos posturas clásicas de las definiciones sociológicas denominadas sustantivistas y funcionalistas (Villamán, 2002).

- La postura sustantivista elige un camino estrecho, restrictivo, muy discriminatorio, evita las ambigüedades, va ligado a la necesidad de un criterio sustantivo de religión. Una figura clave es el inglés Bryan Wilson quien construye un concepto típico-ideal de religión desde el concepto de salvación supraempírica y mundana a la que adscribe dos rasgos principales: la referencia a lo sobrenatural y la eficacia social utópica o capacidad de inspirar y legitimar proyectos de acción orientados a la transformación de la sociedad (Dobbelaere, 2006).

- La postura funcionalista concibe a la religión como vehículo o envoltorio de los universos de significado fundamentales, pluralizados hasta el infinito, que permiten al hombre vivir como individuo social. La debilidad de esta postura es, según Mardones (1998), que al definir la función fundamental de la religión como la construcción social de un universo 
objetivo de significados que tienen un sentido moral permite abrazar cualquier manifestación ideológica moderna.

Se hace indispensable elaborar una propuesta en que se articulen estas dos posturas. En esta línea los sociólogos, en especial D. Hervieu - Léger, propugnan por una definición sustantiva de religión que incluye todo conjunto de creencias y prácticas, más o menos organizadas, referidas a una realidad supraempírica trascendente, y por otra parte ligan su concepto de religión a una serie de funciones limitativas: de integración, identificación, explicación de la experiencia colectiva, respuesta al carácter estructuralmente incierto de la vida individual y social (Hervieu-Léger, 2003). Esta propuesta de mezcla o conjunción de elementos funcionalsustantivos, dentro de un ámbito cultural determinado, con cierto carácter de provisionalidad (Blanchi, 2010), puede llegar a ser la herramienta más adecuada para hacernos cargo de las transformaciones de lo religioso en la sociedad moderna, de sus metamorfosis y manifestaciones (Mardones, 1998). El autor toma posición:

No ocultamos el atractivo que presentan el planteamiento y las hipótesis de D. Hervieu-Léger. Nos parece que afronta con claridad y lucidez la problemática de la modernidad religiosa y que hace esfuerzos coherentes por dar una solución teórica y metodológica a las cuestiones. (Mardones, 1998, p. 45)

Esta propuesta incluye esfuerzos de analogía (también llamados de homología) con las religiones seculares que buscan las relaciones (Aparicio-Gómez, 2006) y correspondencias concretas entre dos actividades, una religiosa y otra secular. Es de tales análisis relacionales y homológicos que se han desprendido muchas anotaciones respecto a la variedad y multiplicidad de las manifestaciones de lo religioso en lo secular.

A nivel general podrá usarse la denominación de sagrado o religión en la modernidad para cualquiera de las manifestaciones metafóricas de la religión (Viale, 2013), aunque es mejor reservar el título de religión para referirse a las que tienen la autoridad de una tradición. Para analizar las formas de lo sagrado en la sociedad moderna actual, los estudios analógicos u 
homológicos explicados arriba para señalar las diversas manifestaciones de lo religioso en lo secular.

\section{Conclusiones}

El tema de lo sagrado puede ampliarse también hablando del totemismo y del animismo.

Importante diferenciar los ritos en los que se busca lo trascendente y lo sagrado, con aquellos que tienen algo de superstición y magia pero que buscan cosas temporales: el horóscopo, las cartas, la interpretación del destino por medio de las estrellas, las hierbas para la buena suerte, etc., pues aquí cabe la pregunta: ¿el creer en cosas sobrenaturales que no se refieren a un "más allá” también entra en el concepto de sagrado o religiosidad?

Indispensable abordar el tema de la religión, su definición, las diferentes teorías sobre la religión de Marx, Weber y Durkheim principalmente, la diferencia entre religión y religiosidad, la diferencia entre la forma de concebir lo trascendente por parte de un individuo de religión monoteísta con la de otro de religión politeísta.

Indispensable abordar los elementos comunes en las sociedades seculares que hacen "sospechar" de la presencia del concepto de sagrado.

Pareciera que Mardones (1998) toma el concepto de secularización como algo que puede ser aplicado a todas las culturas, como si fuera un modelo único hacia el cual tienden o tenderán las sociedades post-modernas. Cabría la pregunta: ¿no será esto una concepción europea?; ¿podrían existir otros tipos de secularización en culturas como la africana o la latinoamericana? Pareciera que el autor tiene cierta tendencia a afirmar que este proceso ha traído algo de positivo a las sociedades y a la misma religión pues les ha dado más libertad y autonomía. 
La concepción cristiana es una barrera muy fuerte a la hora de hacer una crítica sobre si algo es o no una manifestación religiosa.

Importante abordar de una manera adecuada el problema de lo sagrado y sus múltiples manifestaciones, este elemento no desaparece con la secularización, sino que más bien va cambiando su aspecto, va evolucionando con el paso de la cultura y de la historia, pero que, en el fondo, el elemento trascendental hace parte de las personas y de las sociedades ya que es un elemento constitutivamente ontológico del ser.

\section{Referencias}

Abumanssur, E. S. (1970). A arte, a arquitetura e o sagrado. Ciencias Sociales y Religión/Ciências Sociais e Religião. https://doi.org/10.22456/1982-2650.2165

Aparicio-Gómez, Oscar-Yecid (2006). El nexo familia persona en la filosofía personalista (Tesis Doctoral). Barcelona, España: Universidad de Barcelona. Disponible en: http://hdl.handle.net/2445/41696

Aparicio-Gómez, Oscar-Yecid (2016). La persona en la Sociedad Digital. En AparicioGómez, Oscar-Yecid (Eds.). El Uso Educativo de las TIC (pp. 251-256). Bogotá, Colombia: Universidad Central. ISBN: 978-958-26-0316-8

Banners. (2017). Significado de Reacción - Qué es, Concepto y Definición. 17/04/2017.

Barros Cantillo, N. (2015). El Estado Laico. Advocatus. https://doi.org/10.18041/01240102/advocatus. 24.991

Blanchi, I. (2010). Espiritualidad Y Práctica Clínica. APSA.

Diez de Velasco, F. (2011). Conceptos Generales Y Glosario Sobre Religión Y Religiones. Material De Religión. 
Dobbelaere, K. (2006). Bryan Wilson 's contributions to the study of secularization. Social Compass. https://doi.org/10.1177/0037768606064293

Duch, L., Lavaniegos, M., Capdevilla, M., \& Solares, B. (2008). Hombre, Tradición y Modernidad. In Lluís Duch, Antropología Simbólica y Corporeidad Cotidiana.

Durkheim, E. (1968). Definición del fenómeno religioso y de la religión. In Las formas elementales de la vida religiosa.

G., C. P., \& G., C. P. (1994). La sociología de la religión y la modernidad: por una revisión crítica de las categorías durkheimianas desde América Latina. La sociología de la religión y la modernidad: por una revisión crítica de las categorías durkheimianas desde América Latina. Revista Mexicana de Sociología. https://doi.org/10.2307/3541090

Gerth, H. H., Mills, C. W., \& Turner, B. S. (2013). From Max Weber: Essays in Sociology. In From Max Weber: Essays in Sociology. https://doi.org/10.4324/9780203452196

Hervieu-Léger, D. (2003). The role of religion in establishing social cohesion. Religion in the New Europe.

Lamo de Espinosa Michels de Champourcin, E. (2009). Lo sagrado y lo profano. Revista de Occidente.

Lassman, P. (2017). Max weber. In Max Weber. https://doi.org/10.4324/9781315264899

Mardones, José María (1998), Para comprender las nuevas formas de la religión. La reconfiguración postcristiana de la religión. Estella (navarra). Verbo Divino. 
Mundigo, A. I. (2005). Religión y salud reproductiva : encrucijadas y conflictos. II Reunión de Investigación Sobre Embarazo No Deseado y Aborto Inseguro. Desafíos de Salud Pública En América Latina y El Caribe Ciudad de México, 17 a 19 de agosto de 2005.

Paiva, G. J. de, Garcia, A., Gonçalves, A. K., Scala, C. T., Faria, D. G. R. de, Gómez, M. L. T., Jordão, M. P., Barbosa, R. C., \& Franca, S. M. S. (2004). Experiência religiosa e experiência estética em artistas plásticos: perspectivas da psicologia da religião. Psicologia: Reflexão e Crítica. https://doi.org/10.1590/s0102-79722004000200010

Rivera, A. F. (2008). Islas del sol y de la luna: el camino sagrado. The Hebrew University of Jerusalem.

Sánchez Jerez, E. (2007). El mito y la religión. Revista Temas. https://doi.org/10.15332/rt.v0i1.772

Tillich, P. (1987). The shaking of the foundations. Journal of Substance Abuse Treatment. https://doi.org/10.1016/0740-5472(87)90011-0

Viale, C. M. (2013). Sagrado/profano. El aspecto durkheimiano de la filosofía de la religión de William james. Ideas y Valores.

Villamán, M. (2002). La vuelta de lo sagrado: religión y dinámica social. Ciencia y Sociedad. https://doi.org/10.22206/cys.2002.v27i4.pp504-48

Zuidema, R. T. (2020). Mito e historia en el antiguo Perú. Allpanchis. https://doi.org/10.36901/allpanchis.v9i10.845 